\title{
Comparison of linear and logarithmic receiver signals from polarimetric weather radar echoes and their temporal decorrelation properties
}

\author{
P. Tracksdorf ${ }^{1}$, A. Ghorbani ${ }^{1, *}$, M. Chandra ${ }^{1}$, M. Hagen ${ }^{2}$, and D. Bebbington ${ }^{3}$ \\ ${ }^{1}$ Professur für Hochfrequenztechnik und Photonik, Technische Universität Chemnitz, Reichenhainer Strasse 70, \\ 09126 Chemnitz, Germany \\ ${ }^{2}$ Institut für Physik der Atmosphäre, Deutsches Zentrum für Luft- und Raumfahrt (DLR), DLR Oberpfaffenhofen, \\ PF 1116, 82230 Wessling, Germany \\ ${ }^{3}$ University of Essex, Wivenhoe Park, UK - CO4 3SQ, Colchester, UK \\ * visiting faculty member from Amirkabir University of Technology (Tehran Polytechnic), Department of Electrical and \\ Electronic Engineering, Hafez Ave No 424, 15914, Tehran, Iran
}

\begin{abstract}
Usually common polarimetric weather radar DSPproducts (e.g.: reflectivity, differential reflectivity, linear depolarisation ratio - for both - co-polar and cross-polar signal components) are based on the logarithmic receiver output, because of the large dynamic range provided by the logarithmic receiver. In this paper for the first time we also use the linear receiver output to calculate common weather radar DSP-Products. Using the raw time series radar data recorded with the coherent polarimetric C-band weather radar of the DLR ("Poldirad", Wessling, Germany) it is possible to do a comparison between processed weather radar echoes from the linear receiver and the logarithmic receiver. After the comparison showed very good results, we continued the work with the linear receiver data, especially on the topic named temporal decorrelation properties of the linear receiver data. This paper includes the first results obtained from two observables that belong to our working topic. The first observable is the "Time Decorrelation Factor-TDF" and the second one is the "Decorrelation Time DT $\tau$ ". The results have been summarised in the form of empirical relationships, plots and the least mean square (LMS) method of curve fitting was used to give the mathematical relationship for the observables TDF and DT $\tau$. Generally, the paper will also reflect on the statistical properties of radar echoes measured with linear receivers. The usage of the linear receiver data opens a wide field of new applications and products for the work with polarimetric weather radar data, because the linear receiver data also provides phase information which a logarithmic receiver does not.
\end{abstract}

Correspondence to: P. Tracksdorf

(patrick.tracksdorf@s1999.tu-chemnitz.de)

\section{Introduction}

Recent advances in the field of electronics and digital signal processing has let to developments of new modern radar systems and radar components, especially in the fields of receivers, such that these days linear and logarithmic receivers with very low noise and therefore high dynamic range are available to the market. Because the linear receiver output provides magnitude- and phase- information (I/Q-signals) of the received signal, the linear receiver has an advantage to the logarithmic receiver. The logarithmic receiver output consists of the log received power without any phase- information. With increasing digital signal processing power it becomes more and more interesting to use the linear receiver time series data, especially the phase information, to achieve more complex and new weather radar products. Also the common weather radar products can be obtained from the linear receiver output. The complex signal echoes are useful in the area of target identification as well as the target classification and also in the decomposition theories. The coherent polarimetric C-band weather radar of the DLR ("Poldirad", Wessling, Germany) provides two types of receivers working in parallel, as there are: two linear receivers and two logarithmic receivers. One of each type for either the copolar and the cross-polar receiving channel. A schematic block diagram of the Poldirad is given in Fig. 1 and in the following chapter, the Poldirad with its basic specification is introduced. To check the reliability of the linear receiver data of the Poldirad, we performed a comparison of common weather radar products, obtained from both types of receivers. This is possible, because we have archived datasets of the Poldirad, that contain information from all four (two linear and two logarithmic) receivers. 


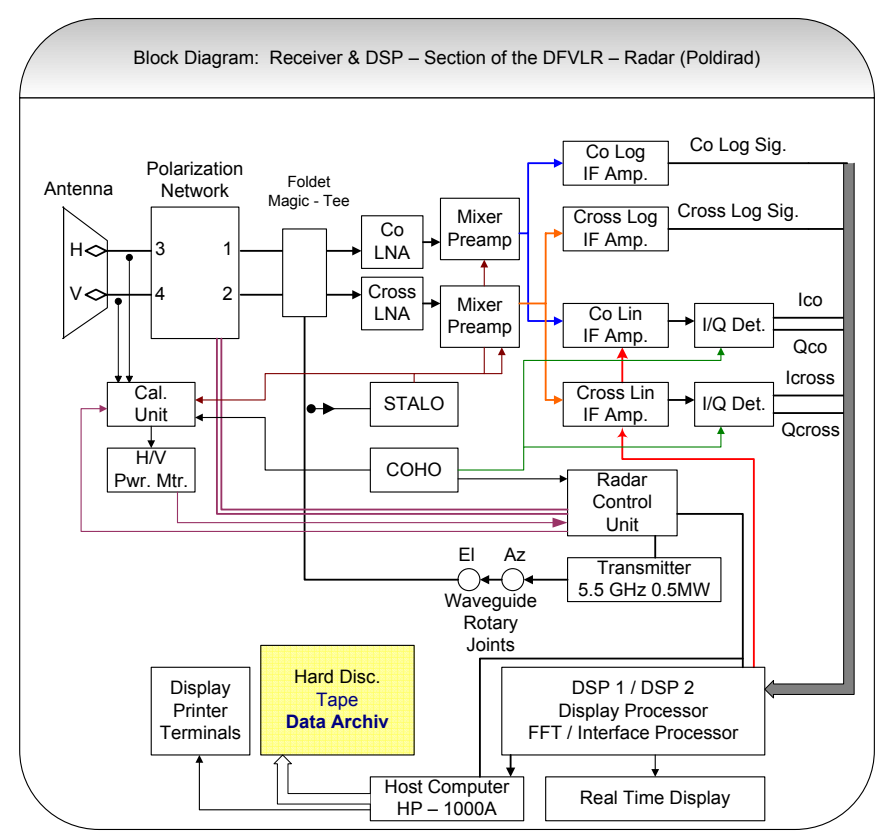

Fig. 1. Block diagram: receiver and DSP-section of the DFVLRRadar "Poldirad".

With the good comparison results, we continued the work with the linear receiver data and started with the topic named "temporal decorrelation properties of linear receiver weather radar data".

The temporal decorrelation properties, especially the decorrelation times of polarimetric weather radar echoes represent an important property from the point of view of measurement accuracy of radar observables and their interpretation. For instance, the magnitudes of decorrelation times have a significant bearing on the choice of pulse repeat frequency and sample size and also in establishing the S-matrix coherence. Despite being a significant parameter in practically all aspects of weather radar applications, it has met with unduly scant attention in the literature to date.

\section{Radar specification and used archive data set}

With refer to Fig. 1 and references Schroth et al. (1988) and Chandra et al. (1992), the specifications of the coherent polarimetric C-Band weather radar "Poldirad" are as follow: It is a C-band radar working with a tuneable frequency from $5.48 \mathrm{GHz}$ to $5.85 \mathrm{GHz}$. There are three possible radar impulse-volume range settings available. The range setting of $300 \mathrm{~m}$ leads to the unambiguous range of $300 \mathrm{~km}$, the range setting of $150 \mathrm{~m}$ leads to the unambiguous range of $120 \mathrm{~km}$ and the high resolution range setting of $75 \mathrm{~m}$ leads to the unambiguous range of $60 \mathrm{~km}$. The maximum available number of range bins is 452 and these range bins can be placed anywhere on the interval from 0 to $900 \mathrm{~km}$, as long as the span from the first to the last range bin is less than $300 \mathrm{~km}$. The dish antenna has an aperture diameter of approximate $5 \mathrm{~m}$ with a focal length of approximate $4 \mathrm{~m}$ and an offset corrugated horn feed. The horizontal beamwidth is $1^{\circ}$ with continuous scan and the vertical beamwidth is also $1^{\circ}$ for the scan from $-6^{\circ}$ to $90^{\circ}$. The gain of the antenna is approximate $44.5 \mathrm{~dB}$ and the side-lobe level of the pattern is less than $-32 \mathrm{~dB}$. The transmitter peak power is $400 \mathrm{~kW}$, the pulse repeat frequency PRF is variable from $160 \mathrm{~Hz}$ to $2400 \mathrm{~Hz}$ (available settings: $160 \mathrm{~Hz}, 400 \mathrm{~Hz}$, $800 \mathrm{~Hz}, 1200 \mathrm{~Hz}$, and $2400 \mathrm{~Hz}$ ) and the available pulse-width settings are $2 \mu \mathrm{s}, 1 \mu \mathrm{s}$, and $0.5 \mu \mathrm{s}$. The loss from the transmitter to the antenna feed is approximate $2.5 \mathrm{~dB}$. The radar has two types of receiver, namely logarithmic and linear receiver. The dynamic range of the logarithmic receiver is $80 \mathrm{~dB}$ and the dynamic range of the linear receiver is $60 \mathrm{~dB}$. There are two receivers from each type available, so that there is a single receiver of each type for co- and cross- polarized receive-channel. The linear receiver has two types of receiver gain control options available, the sensitivity time control (STC) option and the fixed gain option. The STC values are stored in the DSP in form of a look-up table and can be applied to IF amplifier with the range dependence of $1 / \mathrm{R}^{2}$, as shown in Fig. 2. In the fixed gain option, the radar operator has to choose the linear receiver gain reduction in according with the observed weather situation. Such as, in the strong weather situation one has to use lower receiver gain and in weak weather situation the receiver gain has to increase accordingly.

The radar can measure the reflectivity with accuracy less than $\pm 1 \mathrm{~dB}$. The radial velocity and spectral width accuracy is better than $\pm 1 \mathrm{~ms}^{-1}$. The minimum detectable signal is $-108 \mathrm{dBm}$, if a pulse with of $0.5 \mu \mathrm{s}$ is used. In reference Schroth et al. (1988) and Chandra et al. (1992) the radar specification is discussed more detailed. Especially the linear and the logarithmic receiver behaviour is discussed in reference Chandra et al. (1992).

The archived data set used in this work was time series radar data ("Type 18"), which contains data from the linear and the logarithmic receivers for all range bins of the scan.

\section{Theoretical Framework}

The radar provides transmission of general elliptical polarization state $\hat{e}_{1}^{+}$and $\mathrm{T}(\mathrm{T}=1 / \mathrm{PRF}) \mathrm{s}$ later it transmits $\hat{e}_{2}^{+}$orthogonal to $\hat{e}_{1}^{+}$as shown in Fig. 3.

The scan-strategy shown in Fig. 3 is called the S-Matrix measurement mode scheme. This scheme is used to collect the data for time series radar data-type 18. For each transmission, the received co- and cross- polarized echo signals would be amplified by the corresponding low noise amplifier LNA and convert to intermediate frequency IF by the RF-mixer stage. Then the co- polar and cross- polar signal is split and amplified again by IF amplifiers. There are two linear IF amplifiers for the linear receiver section and two logarithmic IF amplifiers for the logarithmic receiver section. After the I/Q detection of the linear receiver signals, the video signals and the log signals are passed to DSP 


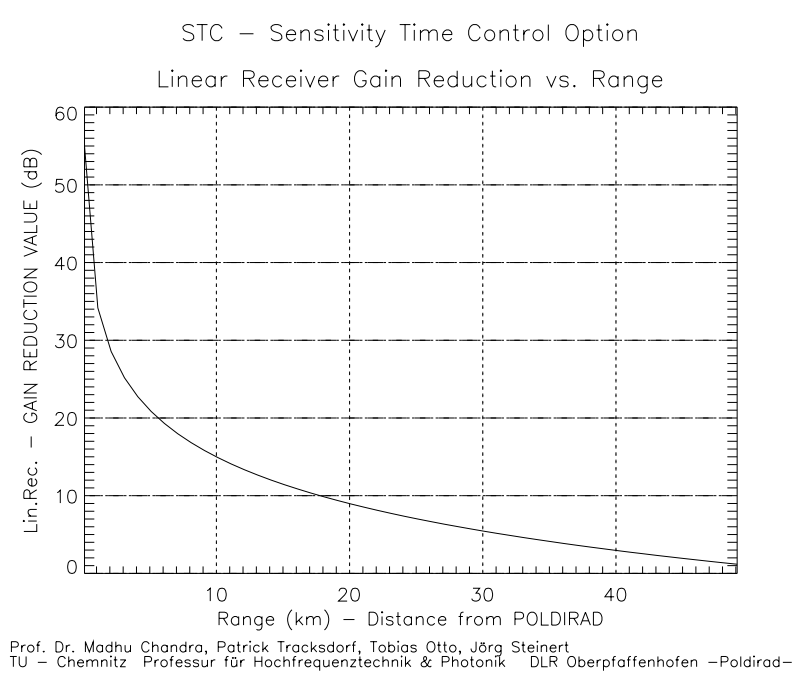

Fig. 2. Sensitivity time control (STC)-option dependence of the linear receiver gain reduction vs. range.

unit for further processing. Please note that the logarithmic receivers work in parallel with the linear receivers, so that there is information from all linear and logarithmic receivers available for each transmitted impulse. One measurement consists of the transmission of two impulses with the two orthonormal transmission states and occurs over a time span of 2T, as shown in Fig. 3. The measurement is repeated sequentially with a predefined number of repetitions $\mathbf{M}(32$, $64,128)$. The results of one measurement with the two defined polarization states are for each transmission state the in-phase and quadrature voltage components from the linear receiver section for co- and cross- channel and for each transmission state the log measured power ( as voltage level ) from the logarithmic receiver section for co- and cross- channel. With this measurement mode scheme, the DSP then computes the results for all bins of the actual ray. A ray includes all range bins of one fixed azimuth and elevation angle. The type 18 datasets we used include the following measurement results for each single range bin of the whole scan.: from the linear receiver section we have the In-phase I and Quadrature-phase Q voltage components (no further processing by the DSP):

$\left(I_{c 1}^{n}, Q_{c 1}^{n}\right),\left(I_{x 1}^{n}, Q_{x 1}^{n}\right),\left(I_{c 2}^{m}, Q_{c 2}^{m}\right)$,

$\left(I_{x 2}^{m}, Q_{x 2}^{m}\right), n=1,3, \ldots, 2 M-1, m=2,4, \ldots, 2 M$

and from the logarithmic receiver section we have the "effective reflectivity factor" (Battan, 1973) - pre processed by the DSP:

$$
\begin{aligned}
& Z_{x x}=10 \log \left(C R^{2} P_{x x}\right)[d B Z](3.1) \\
& Z_{x y}=10 \log \left(C R^{2} P_{x y}\right)[d B Z](3.2) \\
& Z_{y x}=10 \log \left(C R^{2} P_{y x}\right)[d B Z](3.3) \\
& Z_{y y}=10 \log \left(C R^{2} P_{y y}\right)[d B Z](3.4),
\end{aligned}
$$

where $\mathrm{M}$ is the number of measurements, $\mathrm{c} 1$ denotes a co-polar component of the received signal with transmit polarization 1, c2 denotes a co-polar component of the received signal with transmit polarization 2 , x 1 denotes a crosspolar component of the received signal with transmit polarization 1, x2 denotes a cross-polar component of the received signal with transmit polarization $2, \mathrm{Z}$ is the effective reflectivity factor (Battan, 1973) calculated by the DSP with xx .. yy denoting the transmit and receive states (e.g. xy: transmit state 1 receive state 2). Please note $\mathrm{xx}$, yy are the co-polar results of the measurement and xy, yx are the cross-polar results of the measurement. $\mathrm{C}$ denotes the radar constant saved in the DSP and R denotes actual range. With this information for every single range bin and additional information of the whole scan included in the data set type 18 , we can continue with the post processing of the data. From the logarithmic receiver data we can derive some common weather radar parameters, as there are the linear depolarization ratios LDR and the differential reflectivity ZDR. (These definitions are for $\mathrm{H} / \mathrm{V}$-transmit polarization states!)

$$
\begin{aligned}
& L D R_{y x}=10 \log \left(\frac{P_{y x}}{P_{x x}}\right)=Z_{x x}-Z_{y x}[d B](3.5) \\
& L D R_{x y}=10 \log \left(\frac{P_{x y}}{P_{y y}}\right)=Z_{y y}-Z_{x y}[d B](3.6) \\
& Z D R=10 \log \left(\frac{P_{x x}}{P_{y y}}\right)=Z_{y y}-Z_{x x}[d B](3.7)
\end{aligned}
$$

From the linear receiver data the post processing is more complicated. For each measurement we have got eight values available, namely the co- and cross- polar quadrature voltages (I/Q) for each of the two transmit polarization states. For each bin we have got $\mathrm{M}$ measurements leading to a total number of $\mathrm{M} *$ eight values for each bin. The fact that linear receiver time series data lead to a huge amount of data and therefore to a huge amount of processing time and storage place, could be one reason why the linear receiver time series data is not used in common weather radar applications. But with the rapidly increasing processor speed and storage place of modern computers, this fact should be erased. Our starting point were the I and Q values, which were sampled and stored by the DSP. For each range bin we have a series of I and $Q$ values, yielding to a series of in-phase and quadrature voltage components, that can be rearranged to the complex voltages as follows:

$V_{c 1}^{n}=I_{c 1}^{n}+j Q_{c 1}^{n}(3.8)$

$V_{x 1}^{n}=I_{x 1}^{n}+j Q_{x 1}^{n}(3.9)$

$V_{c 2}^{m}=I_{c 2}^{m}+j Q_{c 2}^{m}(3.10)$

$V_{x 2}^{m}=I_{x 2}^{m}+j Q_{x 2}^{m}(3.11)$

$n=1,3, \ldots 2 M-1$

$m=2,4, \ldots, 2 M$,

with $\mathrm{M}$ is the maximum number of impulses per polarization state $(32,64,128)$. With this series of complex voltages we can obtain the S-Matrix elements, because this complex voltages are proportional to the S-Matrix elements. This leads to 


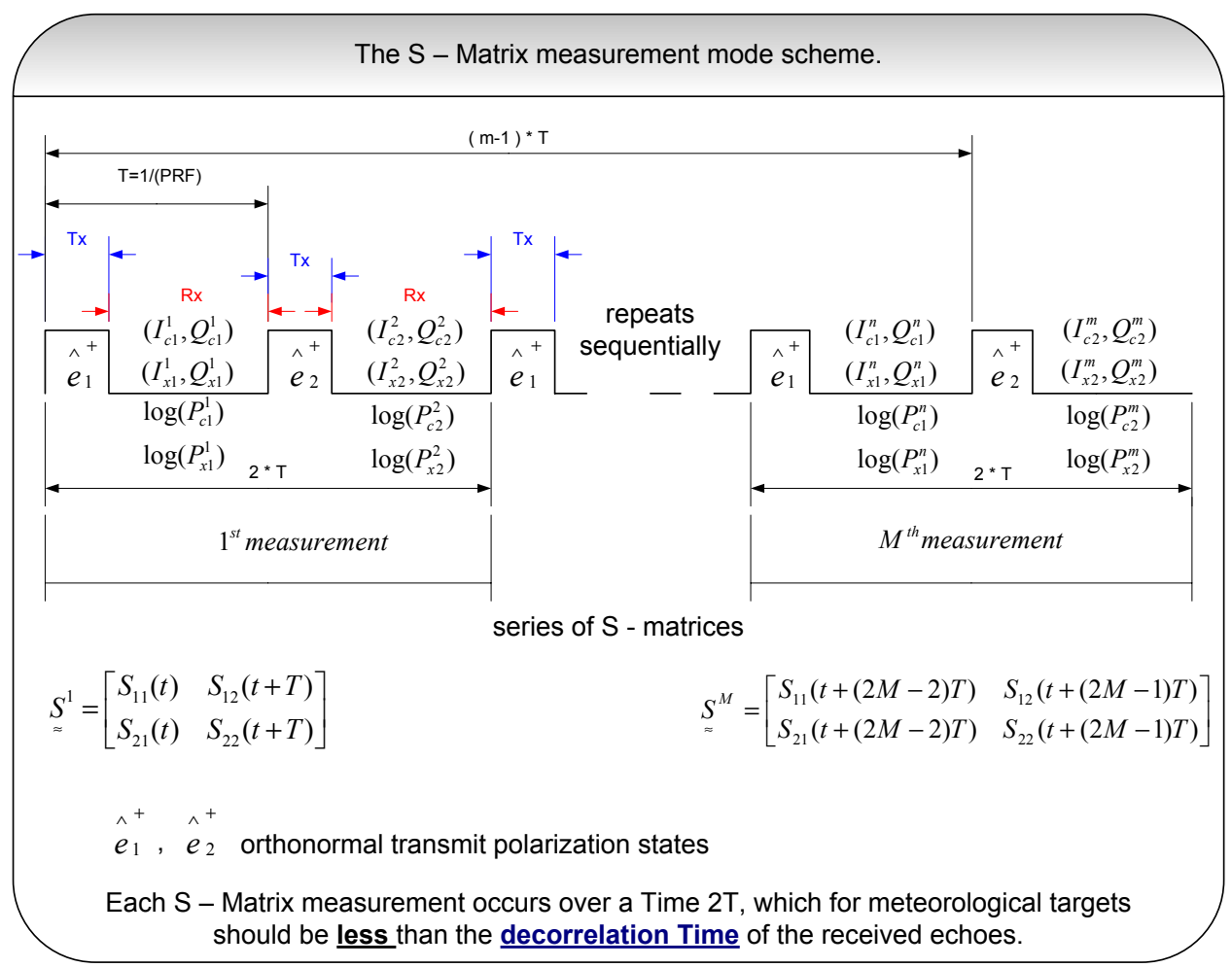

Fig. 3. S-Matrix measurement mode scheme.

a series of S-Matrix elements. By now we ignore the constant in the following formulas and include them at a later stage.

$$
\begin{aligned}
& S_{11}^{n}=\text { const } \cdot\left(V_{c 1}^{n}\right)(3.12) \\
& S_{21}^{n}=\text { const } \cdot\left(V_{x 1}^{n}\right)(3.13) \\
& S_{12}^{m}=\text { const } \cdot\left(V_{x 2}^{m}\right)(3.14) \\
& S_{22}^{m}=\text { const } \cdot\left(V_{c 2}^{m}\right)
\end{aligned}
$$

We now can combine the series of S-Matrix elements to a series of M S-Matrices. After this step of calculation, we can see the time slot of T between the S-Matrix columns, resulting from the S-Matrix measurement mode scheme. This important detail is also visible in Fig. 3. Each S-Matrix measurement occurs over a time span of $2 \mathrm{~T}$, which for meteorological targets should be less than the decorrelation time of the received echoes?

$$
S^{M}=\left[\begin{array}{ll}
S_{11}^{n} & S_{12}^{m} \\
S_{21}^{n} & S_{22}^{m}
\end{array}\right]=\left[\begin{array}{ll}
S_{x x}^{n} & S_{x y}^{m} \\
S_{y x}^{n} & S_{y y}^{m}
\end{array}\right]
$$

With the obtained series of S-Matrices, we can now easily calculate common and new weather radar parameters. Our next step was the calculation of the Reflectivity LZ, the linear depolarization ratios LLDR and the differential Reflectivity LZDR for each range bin of the whole scan, with L denoting that the parameter was derived from the linear receiver measurements.

$$
\begin{aligned}
& L Z_{x x}=10 \log \left(C_{L 1} R^{2}\left\langle S_{x x} S_{x x}^{*}\right\rangle\right)[d B Z] \text { (3.17) } \\
& L Z_{x y}=10 \log \left(C_{L 2} R^{2}\left\langle S_{x y} S_{x y}^{*}\right\rangle\right)[d B Z] \text { (3.18) } \\
& L Z_{y x}=10 \log \left(C_{L 2} R^{2}\left\langle S_{y x} S_{y x}^{*}\right\rangle\right)[d B Z] \text { (3.19) } \\
& L Z_{y y}=10 \log \left(C_{L 1} R^{2}\left\langle S_{y y} S_{y y}^{*}\right\rangle\right) \text { [dBZ] (3.20) } \\
& L L D R 1=10 \log \left(\frac{L Z_{y x}}{L Z_{x x}}\right)[d B](3.21) \\
& L L D R 2=10 \log \left(\frac{L Z_{x y}}{L Z_{y y}}\right)[d B](3.22) \\
& L Z D R=10 \log \left(\frac{L Z_{x x}}{L Z_{y y}}\right)[d B](3.23)
\end{aligned}
$$

Again, LLDR and LZDR is defined for H/V-transmit polarization states. In the formulas 3.17 to 3.20 the $<>$ brackets indicate that the products of the S-Matrix elements is averaged over the number of measurements $\mathrm{M}$. The * denotes the conjugate complex of the $\mathrm{S}$-Matrix element. $\mathrm{R}$ is again the actual range of the range bin. $\mathrm{C}_{L 1}$ and $\mathrm{C}_{L 2}$ are the radar constants. The reason of using two different radar constants is, that we have two linear receivers which are not $100 \%$ equal. In the work this paper is based on, we used only one radar constant $\left(\mathrm{C}_{L 1}=\mathrm{C}_{L 2}\right)$, assuming the two receivers to be equal. For further work we will use two radar constants. The following Eq. (3.24) gives the calculation procedure of the radar constant we used for this work (SIGMET Inc., 2004). The radar constant includes the constant from the Eqs. (3.12 to 
$3.15)$.

$C_{L 1 / 2}=\frac{\lambda^{2} \cdot L_{t} \cdot \operatorname{CONST} T_{1 / 2}}{P_{t} \cdot \tau \cdot \theta \cdot \phi \cdot G^{2}}$

where, $\lambda$-radar wavelength in $\mathrm{cm}, \mathrm{P}_{t}$-transmitted peak power in $\mathrm{kW}, \mathrm{L}_{t}$-transmit loss, $\tau$-pulse width in $\mu \mathrm{s}, \Theta / \phi$ horizontal/vertical half power full beamwidth, antenna gain and CONST includes all calculation constants. With the factor CONST it is possible to have two radar constants, one for co-polar linear receiver channel and one for cross-polar linear receiver channel. With the Eqs. (3.17 to 3.23) we have calculated parameters with the linear receiver data which should be equivalent to the parameters calculated by the DSP from the logarithmic receiver output. The comparison we have done is based on the DSP-products obtained from the logarithmic receiver and the results of the post processing of the linear receiver data (Eqs. 3.17 to 3.20). With the series of S-Matrices we can now create the covariance matrix (Eq. 3.25) as a base for further calculations.

$c=\left[\begin{array}{c}\left\langle S_{x x} S_{x x}^{*}\right\rangle \\ \left\langle S_{y x} S_{x x}^{*}\right\rangle \\ \left\langle S_{x y} S_{x x}^{*}\right\rangle \\ \left\langle S_{y y} S_{x x}^{*}\right\rangle\end{array}\right\rangle\left\langle\begin{array}{l}S_{x x} S_{y x}^{*} S_{y x}^{*} \\ S_{x y} S_{y x}^{*} \\ S_{y y} S_{y x}^{*}\end{array}\right\rangle\left\langle\begin{array}{c}S_{x x} S_{x y}^{*} \\ S_{y x} S_{x y}^{*} \\ S_{x y} S_{x y}^{*} \\ S_{y y} S_{x y}^{*}\end{array}\right\rangle\left\langle\left\langle\begin{array}{c}S_{x x} S_{y y}^{*} \\ S_{y x} S_{y y}^{*} \\ S_{x y} S_{y y}^{*} \\ S_{y y} S_{y y}^{*}\end{array}\right\rangle\right]$

where, $<>$ brackets indicate that the products of the S-Matrix elements (from each S-Matrix of the series of S-Matrices) is averaged over the number of measurements $\mathrm{M}$ and $*$ denotes conjugate complex. Please note, that the elements of the main diagonal are real numbers. With this basis we now continue with the topic of temporal decorrelation properties of weather radar echoes.

Polarimetric measurements deliver S-Matrix measurements, which in general have S-Matrix columns that are decorrelated in time. This occurs because the two columns, depending on the measurement technique, are measure with a time difference of $\Delta t=1 / P R F$, as also illustrated in Fig. 3 . It is therefore important that $\Delta \mathrm{t}$ is smaller than the decorrelation time in order for the measurements to be meaningful. Clearly this issue demands information regarding decorrelation times of precipitation volume, with regard to precipitation intensity, precipitation type and the polarization of the radar echoes. In this contribution we shall consider two categories of parameter that help quantify decorrelation properties. These parameter can be split into complex and real quantities and are defined as followed:

$$
F_{12}(\Delta t)=\frac{\int_{0}^{\infty} f_{1}(t) \cdot f_{2}(t+\Delta t) d t}{\int_{0}^{\infty} f_{1}(t) \cdot f_{2}(t) d t}(3.26)
$$

where, $f_{1}$ and $f_{2}$ are real functions and $\Delta t$ is the time lag. The quantity above thus corresponds to autocorrelation if $\mathrm{f}_{1}=\mathrm{f}_{2}$, otherwise it corresponds to cross-correlation between $\mathrm{f}_{1}$ and $f_{2}$. For easier calculation, the denominator can be chosen to be the autocorrelation of say $f_{1}$. Likewise for the complex case we may define:

$Z_{12}(\Delta t)=\frac{\int_{0}^{\infty} Z_{1}(t) \cdot Z_{2}^{*}(t+\Delta t) d t}{\int_{0}^{\infty} Z_{1}(t) \cdot Z_{2}^{*}(t) d t}$

where, * again denotes conjugate complex. It may be noted that $\mathrm{Z}(\Delta \mathrm{t})$ is now itself complex and thus contains information of relating to amplitude and phase decorrelations separately. It may be important to distinguish between quantities called time decorrelation factor TDF and decorrelation time DT $\tau$. The decorrelation factor TDF is generally defined as:

$T D F=\frac{F_{11}(\Delta t=2 / P R F)}{F_{11}(\Delta t=0)} \cdot 100[\%](3.28)$

For instance as used in the following Eqs. (3.29 to 3.32):

$$
\begin{aligned}
& T D F_{x x}=\frac{\left|\left\langle S_{x x}^{*}(t) S_{x x}(t+2 / P R F)\right\rangle\right|}{\left|\left\langle S_{x x}^{*}(t) S_{x x}(t)\right\rangle\right|} \cdot 100[\%](3.29) \\
& T D F_{x y}=\frac{\mid\left\langle S_{x y}^{*}(t) S_{x y}(t+2 / P R F)\right|}{\mid\left\langle S_{x y}^{*}(t) S_{x y}(t)\right|} \cdot 100[\%](3.30) \\
& T D F_{y x}=\frac{\mid\left\langle S_{y x}^{*}(t) S_{y x}(t+2 / P R F)\right|}{|| S_{y x}^{*}(t) S_{y x}(t) \mid} \cdot 100[\%](3.31) \\
& T D F_{y y}=\frac{\mid\left\langle S_{y y}^{*}(t) S_{y y}(t+2 / P R F)\right|}{\mid\left\langle S_{y y}^{*}(t) S_{y y}(t)\right|} \cdot 100[\%](3.32)
\end{aligned}
$$

We can calculate the time decorrelation factor for each element of the S-Matrix. It may be noted, that in the Eqs. (3.29 to 3.32) the $<>$ brackets indicate that the products of the SMatrix elements have to be averaged. In contrast the decorrelation time DT $\tau$ is given as the value of $\Delta t$, here named as $\tau$ for which: (with $\mathrm{e}=2.718281$ Euler constant)

$\frac{F_{11}(\Delta t=\tau)}{F_{11}(\Delta t=0)}=e^{-1}(3.33)$

It may be noted, that we can define three types of decorrelations, the first is magnitude decorrelation, second is power decorrelation and the third is the phase decorrelation. For these three types, we can calculate a decorrelation time. Figure 4 shows an example for the Eq. (3.33).

As we can see from Fig. 4 the decorrelation time DT $\tau$ can be found by calculating the correlation values for a couple of time lags (or easier all time lags) and then we have to determine the time $\tau[\mathrm{s}]$, where the correlation value drops below the value of $\mathrm{e}^{-1}$. It could happen, that due to noise, the correlation value does not drop below the mentioned value. In this case we can take the stable descending part of the correlation value curve (Fig. 4) an do a linear approximation, ignoring the unstable part of the curve. A very similar procedure can be applied to the complex case, but we have to be careful with the definition of decorrelation time, especially when working with phase-decorrelation times. A theoretical assessment of decorrelation effects beg to consider the influence of the following factors: 


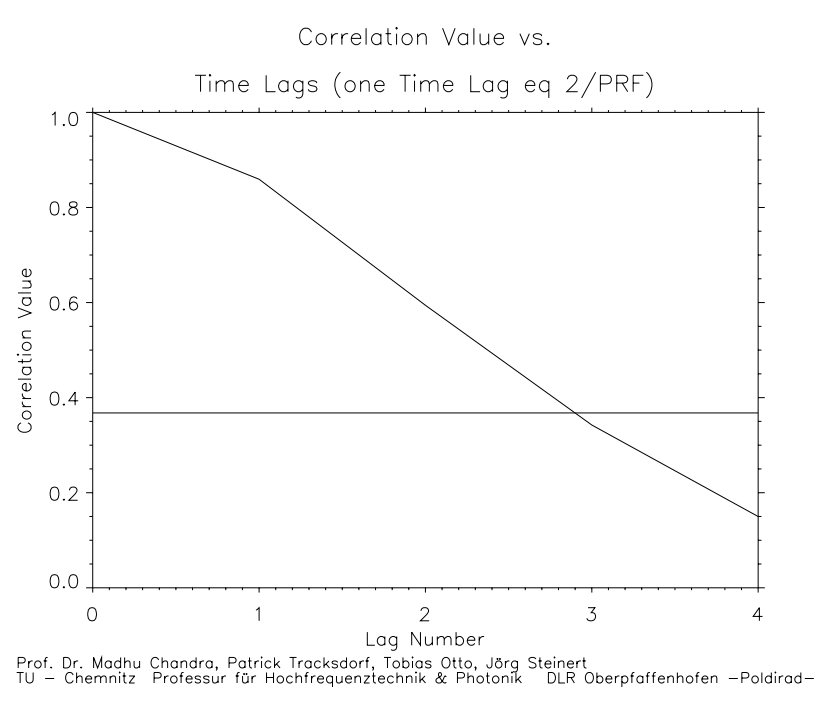

Fig. 4. Correlation value vs. time lags (1 lag=2/PRF [s]).

(1): the mean life time of a particle before it breaks up or collides

(2): the velocity distribution of the particles in the impulse volume

(3): the turbulence in the impulse volume that imparts a random component in the velocities and last but not least the antenna rotation

At this stage of our work, we are making a quantitative assessment of the collective influence of all these aspects. With the exception of antenna rotation. This is true, because the data used was recorded with very slow antenna scanning speeds.

\section{Results}

First a few words to the database we have used. We have used archived datasets from the coherent polarimetric C-band weather radar "Poldirad" in Wessling (Germany), from the years 1987 to 1998 . The available datasets cover all possible variations of the radar parameters like, pulse repetition frequency, pulse width, transmitted power, polarization bases and sample size. The basic specifications of the polarimetric weather radar "Poldirad" are given in Sect. 2. In this section we present the first results of our work, starting with the results from the comparison of linear and logarithmic receiver signals of polarimetric weather radar echoes, followed by the results from the topic of temporal decorrelation properties of weather radar echoes. The results will be presented as figures or tables with a short description in the following two Sects. 4.1 and 4.2. The discussion and interpretation of the results follows in Sect. 5 .
4.1 Comparison of linear and logarithmic receiver signals of weather radar echoes

This section includes the results from the comparison of linear and logarithmic receiver signals of polarimetric weather radar echoes. The results are presented in the Figs. 5, 6, 7 and 8. All these four figures are scatter-plots, where common weather radar observables calculated from the logarithmic receiver signals by the DSP are plotted against the corresponding weather radar observables we have calculated from the linear receiver signals. In each of the four plots, the $\mathrm{x}$ axis is related to the observable obtained from the linear receiver signals and the $y$-axis is related to the observable obtained from the logarithmic receiver signals. All four plots also include a " $\mathrm{x}=\mathrm{y}$ "-line, so that a visual interpretation of the scatter-plots is possible. Figures 5 and 6 show the comparison results for the weather radar observable "Reflectivity", where Fig. 5 shows the result for the co-polar receiver channel and Fig. 6 shows the result for the cross-polar receiver channel. Figure 7 shows the comparison results for the weather radar observable "Linear Depolarization Ratio 2" and last but not least Fig. 8 shows the result for the weather radar observable "Differential Reflectivity". The calculation procedures for the weather radar observables were given in the previous section.

\subsection{Temporal decorrelation properties of weather radar echoes}

In this section we present our first results from the topic "temporal decorrelation properties of weather radar echoes". The results are shown in Figs. 9, 10, 11, 12 and in the Tables 1 and 2. The four figures are again scatter-plots, where temporal decorrelation properties of weather radar echoes are plotted against the weather radar observable "Reflectivity". All the observables in these four plots are obtained from the linear receiver signals as described in Sect. 3. The four plots have been curve fitted with the "least mean square"-method of curve fitting to give a mathematical relationship between the plotted observables. The curve fitting for each of the four scatter-plots is presented, explained and discussed in Sect. 5. Figure 9 shows the time decorrelation factor "TDF" obtained from the co-polar linear receiver channel plotted against the corresponding reflectivity from the co-polar linear receiver channel. The following Figs. 10 and 11 are both representing the same data, but the curve fitting in the two plots is different. Both figures show the decorrelation time "DT $\tau$ xy" obtained from the cross-polar linear receiver channel plotted against the corresponding reflectivity. Related to Figs. 10 and 11 is the Table 2 . Table 2 contains empirical relationships between the decorrelation time "DT $\tau$ xy" and defined weather regions. Figure 12 shows the decorrelation time "DT $\tau$ xx" obtained from the co-polar linear receiver channel plotted against the corresponding reflectivity. Related to Fig. 12 is the Table 1, which again contains empirical relationships between the decorrelation time and defined weather regions, but now for the co-polar linear receiver channel, so 


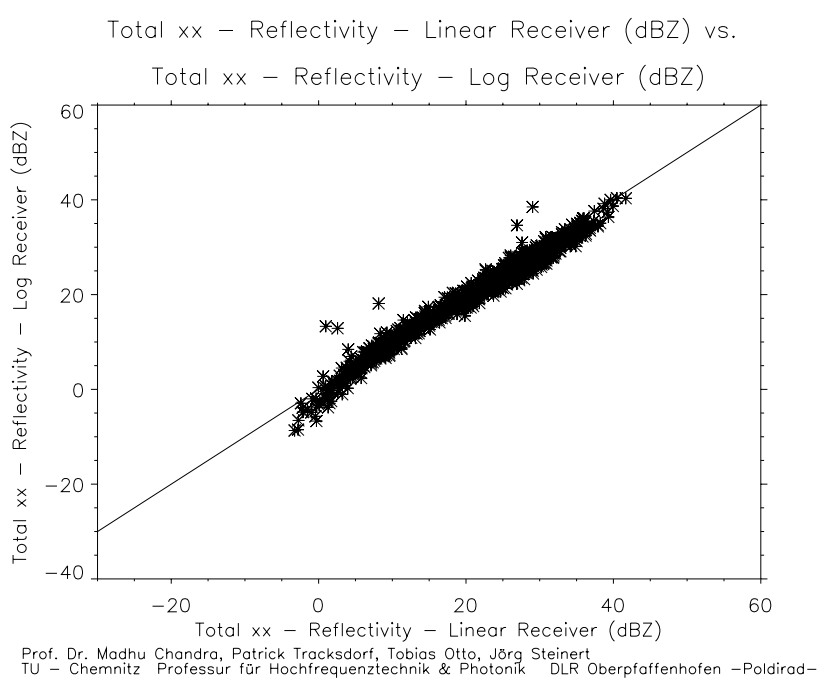

Fig. 5. Reflectivity calculated with linear receiver data (x-axis) vs. Reflectivity calculated by DSP with logarithmic receiver data (yaxis) the solid line: $\mathrm{x}=\mathrm{y}$ line; result from the "co-polar receiver"; Basis transmit: $\mathrm{V}$, receive: $\mathrm{V}$.

the presented observable is "DT $\tau \mathrm{xx}$ ". The calculation procedures for the presented weather radar observables are again given in the Sect. 3. For completeness we can give the defined weather region as follows. In Figs. 9, 10, 11 and 12 we distinguish between the "melting band" and the "normal weather region", where the "normal weather region" can be split into the "weak weather situation" and the "strong weather situation". In Tables 1 and 2 we distinguish between the "melting band" and again the "normal weather region", but this time the "normal weather region" is split into "rain situation", "snow situation" and "very weak weather situation". The defined weather regions are also discussed in the following section.

\section{Discussion of the Results}

In this section we discuss the results from the Sects. 4.1 and 4.2 , starting again with the comparison of linear and logarithmic receiver signals from polarimetric weather radar echoes. The results are shown in the Figs. 5, 6, 7 and 8. These four figures are obtained from one dataset. In Figs. 5 and 6 the reflectivity calculated from logarithmic receiver signal by the DSP is plotted against the reflectivity we calculated from linear receiver signals. Figure 5 shows the result for the co-polar receiver channel and Fig. 6 shows the result for the cross-polar receiver channel. As we can see from Figs. 5 and 6 , there is a very good agreement between the reflectivities calculated from logarithmic receiver signals by the DSP and reflectivities calculated from linear receiver signals. The solid line in Figs. 5 and 6 is the $x=y$ line. The result for the co-polar receiver channel (Fig. 5) is more accurate then the result for the cross-polar receiver channel (Fig. 6). That's due to the fact, that we used the same radar constant (Eq. 3.24)

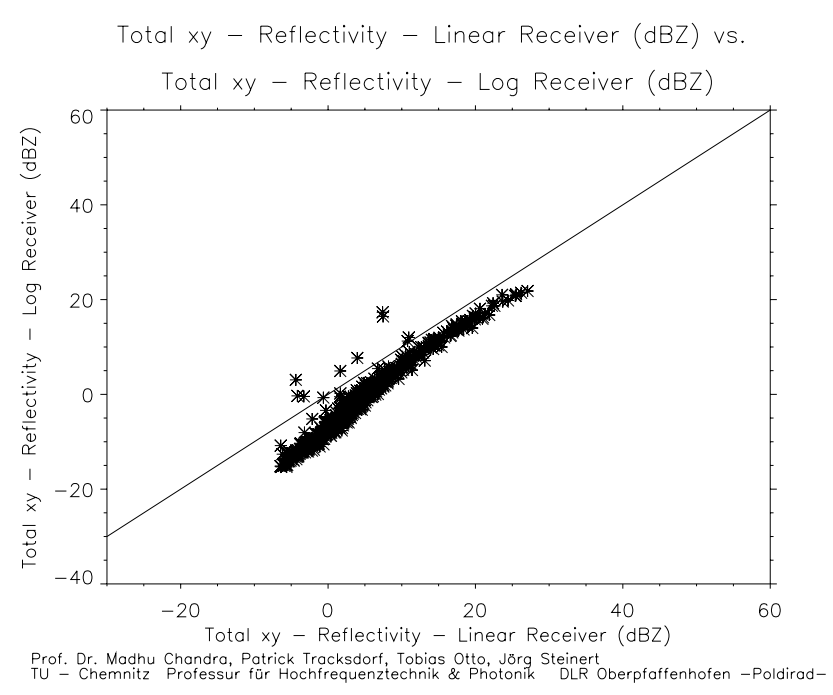

Fig. 6. Reflectivity calculated with linear receiver data (x-axis) vs. Reflectivity calculated by DSP with logarithmic receiver data (yaxis) the solid line: $\mathrm{x}=\mathrm{y}$ line; result from the "cross-polar receiver"; Basis transmit: $\mathrm{V}$, receive: $\mathrm{H}$.

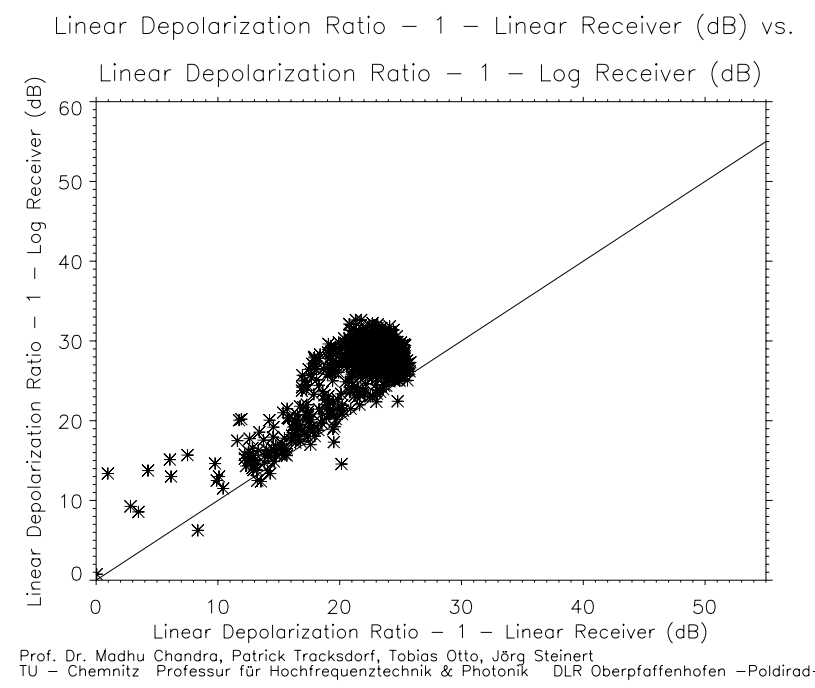

Fig. 7. LDR2 calculated with linear receiver data (x-axis) vs. LLDR2 calculated with logarithmic receiver data (y-axis) the solid line: $\mathrm{x}=\mathrm{y}$ line; result from both co- and cross-polar receivers (see definition Eqs. 3.6, 3.22); Basis transmit: $\mathrm{V} / \mathrm{H}$, receive: $\mathrm{V} / \mathrm{H}$.

$\left(\mathrm{CONST}_{1}=\mathrm{CONST}_{2}\right)$ for both linear receiver channels. So there must be a small difference, because the two linear receivers (co- and cross- linear receiver) are not $100 \%$ the same. In our future work we will use two radar constants, one for each linear receiver channel. We have done this comparison with several datasets between 1987 and 1998 and it turned out that the radar constant, and so the radar hardware (transmitter, receiver, antenna) itself, is changing over the years. That's due to the fact that the DLR maybe has changed broken parts of the radar or that there are some parts having aging effects. Figure 7 shows the comparison of the 


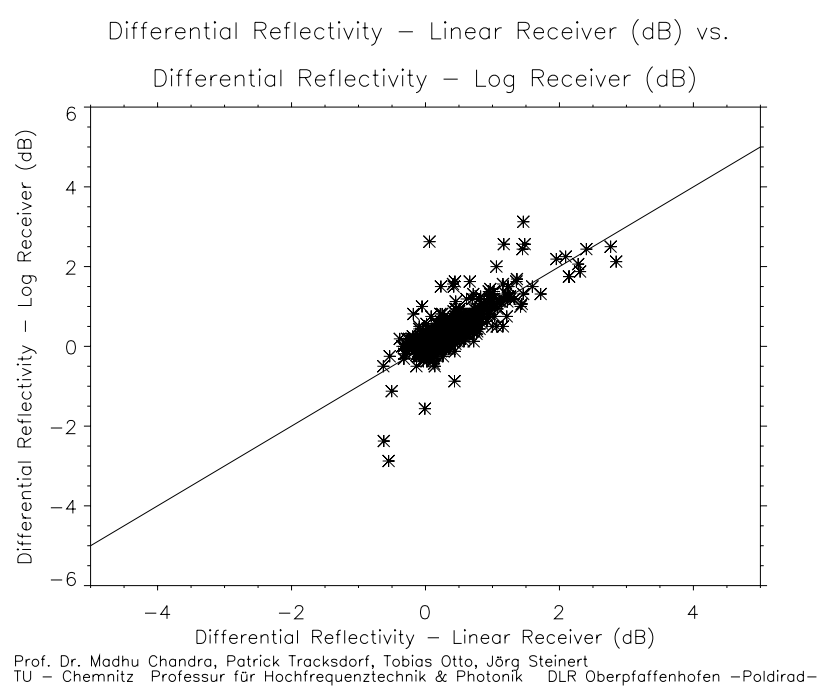

Fig. 8. LZDR calculated with linear receiver data (x-axis) vs. ZDR calculated with logarithmic receiver data (y-axis) the solid line: $\mathrm{x}=\mathrm{y}$ line; result from both co-polar and cross-polar receivers Basis transmit: $\mathrm{V} / \mathrm{H}$, receive: $\mathrm{V} / \mathrm{H}$.

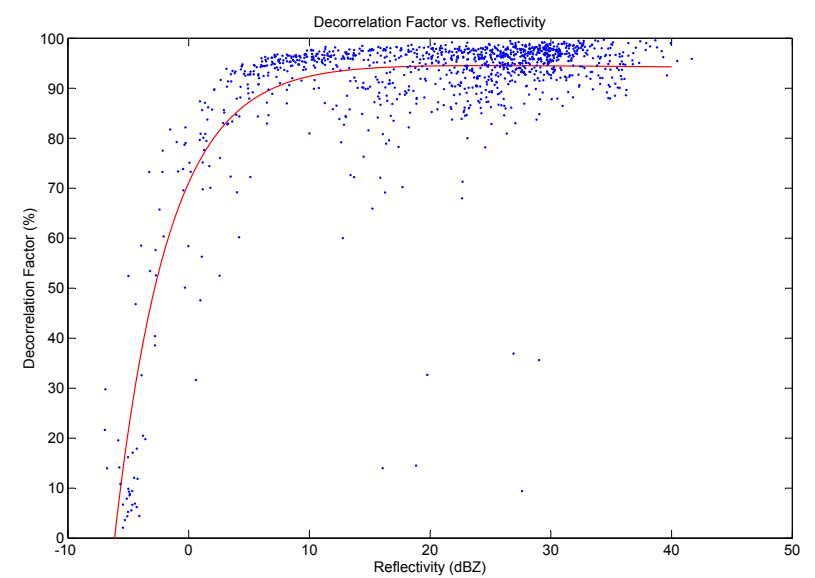

Fig. 9. Time decorrelation factor $\mathrm{TDF}_{x x}$ (y-axis) vs. Reflectivity (x-axis) red solid line - curve fit of the blue data points Basis transmit: V, receive: V; PRF: $1200 \mathrm{~Hz}$; PW: $1 \mu$ s; Sample Size: 128.

linear depolarization ratio LDR2 calculated from logarithmic receiver signals with LLDR2 calculated from linear receiver signals. Again there is a good agreement between the observables LDR2 and LLDR2 calculated from logarithmic and linear receiver signals. Figure 8 shows the comparison for the differential reflectivity ZDR, again with a good agreement. This observation is consistent with the statistical properties of the linear and logarithmic radar echoes. With the results in Figs. 5, 6, 7 and 8 we have done the comparison of linear and logarithmic receiver signals for common weather radar observables with good agreement. We can conclude, that the linear receiver data is accurate in comparison to the logarithmic receiver data and that we can use it for weather radar data

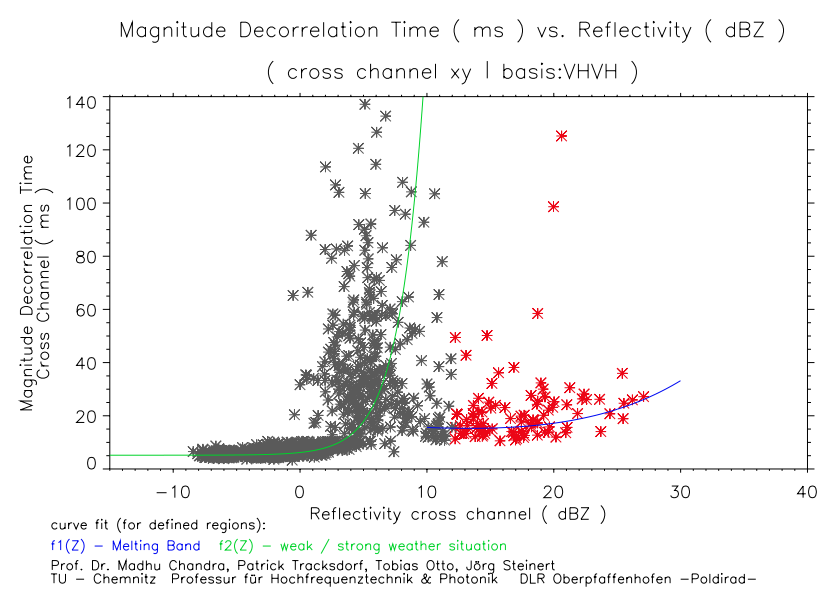

Fig. 10. Decorrelation Time DT $\tau_{x y}$ (y-axis) vs. Reflectivity (x-axis) Basis transmit: V, receive: H; PRF: $1200 \mathrm{~Hz}$; PW: $1 \mu \mathrm{s}$; Sample Size: 128 , red data points belong to the melting band/solid lines: curve fit of plot.

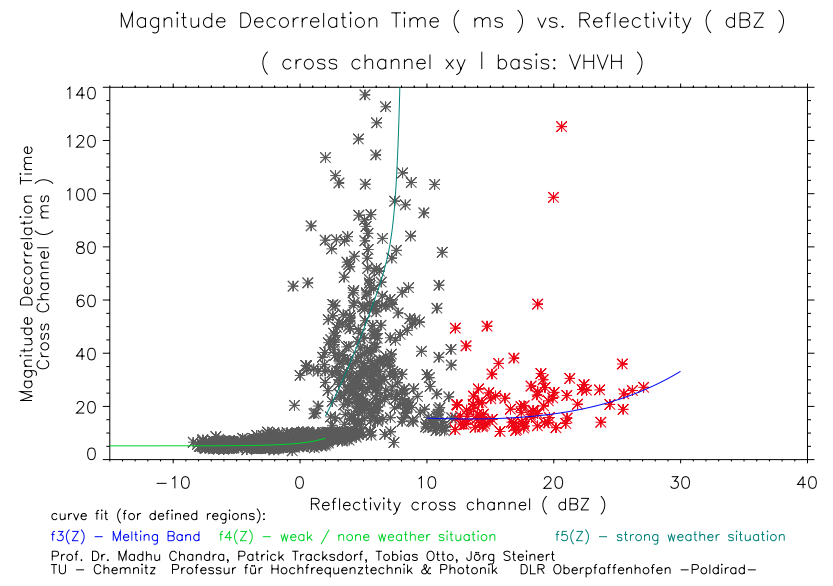

Fig. 11. Decorrelation Time DT $\tau_{x y}$ (y-axis) vs. Reflectivity (x-axis) Basis transmit: V, receive: H; PRF: $1200 \mathrm{~Hz}$; PW: $1 \mu \mathrm{s}$; Sample Size: 128 , red data points belong to the melting band/solid lines: curve fit of plot.

analysis. The fact, that the linear receiver data also provides phase information, opens a wide field for new approaches in weather radar data analysis.

Now we continue with discussion of the results from the topic temporal decorrelation properties of polarimetric weather radar echoes. The results are shown in Figs. 9, 10, 11, 12 and in the Tables 1 and 2. In Fig. 9 we can see the time decorrelation factor TDF plotted against the reflectivity, for the co-polar channel. We have used the least mean square method (LMS) of curve fitting to find the best fit between the two observables. The curve fitting can be seen in Fig. 9 as a solid red line. The following formula gives the result from curve fitting as an exponential curve: 


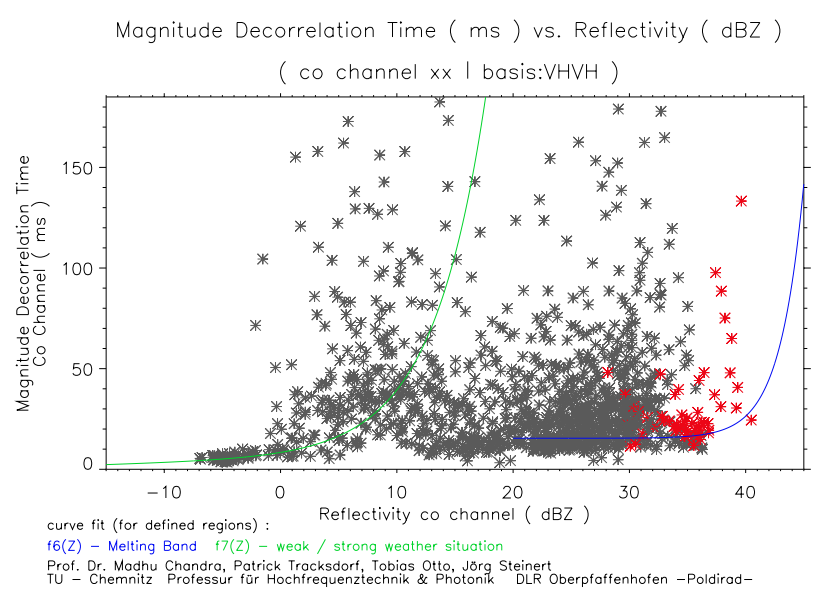

Fig. 12. Decorrelation Time DT $\tau_{x x}$ (y-axis) vs. Reflectivity (x-axis) Basis transmit: V, receive: V; PRF: $1200 \mathrm{~Hz}$; PW: $1 \mu \mathrm{s}$; Sample Size: 128 , red data points belong to the melting band/solid lines: curve fit of plot

$f\left(Z_{x x}\right)=95.17 * \exp \left(-0.0002341 * Z_{x x}\right)$

$-24.07 * \exp \left(-0.2242 * Z_{x x}\right)(5.1)$

For small reflectivities ( $-10 \mathrm{dBZ}$ to $5 \mathrm{dBZ}$ ) the time decorrelation factor TDF is increasing very rapidly and for higher reflectivities $(5 \mathrm{dBZ}$ to $40 \mathrm{~dB})$ the time decorrelation factor TDF is not stationery but very slowly increasing to $100 \%$. Small reflectivities indicate a very weak weather situation with few moving particles in the observed range bin and therefore the two pulse lag $(\Delta \mathrm{t}=2 / \mathrm{PRF})$-correlation value will be small. Also the noise takes place in a weak weather situation, which has a negative influence on the two pulse lag correlation value. With increasing number of moving particles (stronger weather situation) the reflectivity will increase. The random media in the observed range bin has now more reflecting particles, that leads to the result, that the noise loses its influence and that the two pulse lag-correlation value will be stronger. In strong weather situation the range bin is randomly filled with lots of moving particles, that lead to a strong two pulse lag-correlation value, which is increasing slowly to $100 \%$ with increasing reflectivity. These considerations also suggest that stronger rain volumes, apart from having more numerous raindrops in the active volume, also have a more monodisperse distribution of the particles.

In Figs. 10 and 11 we can see the decorrelation time DT $\tau_{x y}$ from the cross-polar linear receiver channel plotted against the reflectivity from the cross-polar linear receiver channel. The used transmit polarization state was vertical and the receive polarization state was horizontal. The Figs. 10 and 11 show the same data but the application of the curve fitting is different. These plots were obtained with the real calculation method (Eq. 3.26) and this decorrelation time is based on magnitude. Therefore we can give the formula for $f_{1}$ and $\mathrm{f}_{2}$ as follows:

$f_{1}(t)=f_{2}(t)=\left(\left|S_{x y}(t)\right|\right)(5.2)$

and complete the name to magnitude decorrelation time $\operatorname{mDT} \tau_{x y}$. The same definition for $\mathrm{f}_{1}$ and $\mathrm{f}_{2}$ was also used for the basis of Table 2. From Figs. 10 and 11 we can see that for very small reflectivities ( $-10 \mathrm{dBZ}$ to $2 \mathrm{dBZ}$ ) the calculated decorrelation times are small and increasing slowly. Small reflectivities are again related to weak weather situation, the random media is filled with few moving particles and the noise gains influence. Here, the movement of the few particles have a larger effect on the correlation value. That will cause the correlation value to drop fast below the value of $\mathrm{e}^{-1}$ and therefore the decorrelation time will be smaller. For higher reflectivities ( $2 \mathrm{dBZ}$ to $11 \mathrm{dBZ}$ ) the decorrelation times are increasing very rapidly. This behavior is as expected, because in strong weather situations (higher reflectivities) the random media is randomly filled with lots of moving particles, noise loses its influence and therefore the decorrelation times should be higher. The movement of the many particles have a smaller effect on the correlation value and it drops slower below the value of $\mathrm{e}^{-1}$. The red data points in Figs. 10 and 11 belong to the melting band. We can see that the data points which belong to the melting band have high reflectivity values ( $11 \mathrm{dBZ}$ to $28 \mathrm{dBZ}$ ) but significantly smaller magnitude decorrelation times. For the melting band we can say that it is more turbulent and therefore the decorrelation times, even if the reflectivities are stronger, are smaller then in other regions with high reflectivities. We have used the least mean square method (LMS) of curve fitting to find the corresponding mathematical relationships between the magnitude decorrelation time and reflectivity for the defined regions. As a first step (Fig. 10) we decided to distinguish between the melting band and the "normal weather region" (which includes weak and strong weather situations). The curve fitting for the melting band can be seen in Fig. 10 as a blue solid line. The following formula gives the result from curve fitting for the melting band as an exponential curve:

$f_{1}\left(Z_{x y}\right)=-33.5275 * \exp \left(0.04311 * Z_{x y}\right)$

$+14.6644 * \exp \left(0.0694 * Z_{x y}\right)+37.8688(5.3)$

The curve fitting for the "normal weather region" can be seen in Fig. 10 as a light green solid line. The following formula gives the result from curve fitting for the "normal weather region" as an exponential curve:

$f_{2}\left(Z_{x y}\right)=1.3814 * \exp \left(0.5036 * Z_{x y}\right)$

$-0.3583 * \exp \left(0.5036 * Z_{x y}\right)+5.1783(5.4)$

As a next step (Fig. 11), we decided to distinguish between the melting band, weak weather situation and strong weather situation. Again, the curve fitting for the melting band can be seen in Fig. 11 as a blue solid line. The following formula 
gives the result from curve fitting for the melting band as an exponential curve: (same as Eq. 5.3)

$$
\begin{aligned}
& f_{3}\left(Z_{x y}\right)=-33.5275 * \exp \left(0.0431 * Z_{x y}\right) \\
& +14.6644 * \exp \left(0.0694 * Z_{x y}\right)+37.8688(5.5)
\end{aligned}
$$

The curve fitting for the "weak weather region" can be seen in Fig. 11 as a green solid line. The following formula gives the result from curve fitting for the "weak weather region" as an exponential curve:

$$
\begin{aligned}
& f_{4}\left(Z_{x y}\right)=2.5269 * \exp \left(0.5284 * Z_{x y}\right) \\
& -1.4995 * \exp \left(0.5384 * Z_{x y}\right)+5.1798(5.6)
\end{aligned}
$$

The curve fitting for the "strong weather region" can be seen in Fig. 11 as a dark green solid line. The following formula gives the result from curve fitting for the "strong weather region" as an exponential curve:

$$
\begin{aligned}
& f_{5}\left(Z_{x y}\right)=(1.72715 e-10) * \exp \left(3.3579 * Z_{x y}\right) \\
& -88.2583 * \exp \left(0.0895 * Z_{x y}\right)-89.2516(5.7)
\end{aligned}
$$

In Fig. 12 we can see the decorrelation time DT $\tau_{x x}$ from the co-polar linear receiver channel plotted against the reflectivity from the co-polar linear receiver channel. The used transmit polarization state was vertical and the receive polarization state was vertical (co-polar). This plot was also obtained with the real calculation method Eq. (3.26) and this decorrelation time is based on magnitude. For the co-polar case we can give the formula for $\mathrm{f}_{1}$ and $\mathrm{f}_{2}$ as follows:

$$
f_{1}(t)=f_{2}(t)=\left(\left|S_{x x}(t)\right|\right)(5.8)
$$

and complete the name to magnitude decorrelation time $\operatorname{mDT} \tau_{x x}$. The same definition for $\mathrm{f}_{1}$ and $\mathrm{f}_{2}$ was also used for the basis of Table 1. The red data points in Fig. 12 belong to the melting band. Again we distinguished between the melting band and the "normal weather region". The curve fitting for the "melting band" can be seen in Fig. 12 as a dark blue solid line. The following formula gives the result from curve fitting for the "melting band" as an exponential curve:

$$
\begin{aligned}
& f_{6}\left(Z_{x x}\right)=(-3.2734 e-11) * \exp \left(0.4494 * Z_{x x}\right) \\
& +(3.4861 e-8) * \exp \left(0.4892 * Z_{x x}\right)+15.43(5.9)
\end{aligned}
$$

The curve fitting for the "normal weather region" can be seen in Fig. 12 as a green solid line. The following formula gives the result from curve fitting for the "normal weather region" as an exponential curve:

$$
\begin{aligned}
& f_{7}\left(Z_{x x}\right)=3.6459 * \exp \left(0.2202 * Z_{x x}\right) \\
& -332.2287 * \exp \left(-0.00051 * Z_{x x}\right)+336.9638(5.10)
\end{aligned}
$$

The wide scatter of the data points in Fig. 12 reflects the need to consider separating the points further, i.e., on the basis of weather type, range, power level and doppler spread.
Tables 1 and 2 contain the empirical results from several datasets for magnitude decorrelation time vs. reflectivity in predefined regions. We decided to split the results for copolar channel results (Table 1) and cross-polar channel results (Table 2). The regions have been defined with the help of the related reflectivity-plot (the whole dataset). We collected empirical results for two polarization bases and for each of the two polarization bases we decided to take two different range resolutions (basis notation: transmit1, transmit2, receive1, receive2). What can be seen as a whole from Tables 1 and 2 is, that with increasing range resolution (and increasing PRF) the magnitude decorrelation times also increase. In region "none" with weak weather situation or no weather situation at all (very small reflectivities), it can be seen, that the magnitude decorrelation times are small. The highest magnitude decorrelation times appear in the rain and heavy rain situation. From Tables 1 and 2 it can be seen, that the magnitude decorrelation times for the melting band are smaller than the magnitude decorrelation times for the rain and heavy rain situation (although the reflectivities in the melting band are higher). There is a small difference in magnitude decorrelation times for different polarization bases.

\section{Conclusion}

In this paper, we used the linear receiver data measured with the coherent polarimetric C-band weather radar "Poldirad" to calculate common weather radar observables and the covariance matrix. From the covariance matrix elements, the reflectivity LZ, the linear depolarization ratios LLDR $1 / 2$ and the differential reflectivity LZDR were estimated for linear receiver. These data were compared with the reflectivity $\mathrm{Z}$, the linear depolarization ratios LDR1/2 and the differential reflectivity ZDR obtained from the pre-processed (by the DSP) logarithmic receiver data. It was observed that there is a good agreement between the corresponding observables, which validates the procedure we used and also indicates that the linear receiver data is as reliable as the logarithmic receiver data. Furthermore, the linear receiver data also provides phase-information of the received signal, this is not the case with logarithmic receiver data. The phase information of the signal is an important issue in polarimetric investigation. Also in this paper, using the linear receiver data, the time decorrelation factor was estimated and plotted vs. the reflectivity. This plot has then been curve fitted to give the corresponding mathematical relationship. As a next step, the decorrelation times were estimated and the magnitude decorrelation time was plotted vs. the reflectivity (separately for the co-polar and the cross-polar receive channel). These plots have then been curve fitted to give the corresponding mathematical relationship for defined regions. For the magnitude decorrelation time we also have collected empirical results for defined regions from various scans and created two tables combining these results for co-polar and cross-polar linear receiver channel independently. These empirical results cover scans with different polarization bases and different 
Table 1. Empirical results: Decorrelation Time DT $\tau_{x x}$ for defined regions, results from co-polar linear receiver channel; real calculation method multiple scans as empirical basis; res. = range resolution.

\begin{tabular}{|c|c|c|c|c|c|c|}
\cline { 4 - 7 } \multicolumn{2}{c|}{} & \multicolumn{4}{c|}{ Decorrelation Time [ms] for region } \\
\hline PRF $[\mathrm{Hz}]$ & res. [m] & Basis ttrr & meltingband & rain & snow & none \\
\hline 1200 & 150 & VHVH & $10 . .20$ & $21 . .>40$ & $>20$ & n.a. \\
\hline 400 & 300 & VHVH & $10 . .18$ & $18 . .>40$ & $20 . .40$ & $<10$ \\
\hline 1200 & 150 & LRLR & $13 . .20$ & $22 . .>40$ & $>20$ & $<10$ \\
\hline 400 & 300 & LRLR & $10 . .15$ & $15 . .>40$ & $18 . .20$ & $<10$ \\
\hline
\end{tabular}

Table 2. Empirical results: Decorrelation Time DT $\tau_{x y}$ for defined regions, results from cross-polar linear receiver channel; real calculation method multiple scans as empirical basis; res. = range resolution.

\begin{tabular}{|c|c|c|c|c|c|c|}
\cline { 4 - 7 } \multicolumn{2}{c|}{} & \multicolumn{4}{c|}{ Decorrelation Time [ms] for region } \\
\hline PRF [Hz] & res. [m] & Basis ttrr & meltingband & rain & snow & none \\
\hline 1200 & 150 & VHVH & $10 . .20$ & $10 . .>40$ & $. .20 .$. & $<5$ \\
\hline 400 & 300 & VHVH & $10 . .15$ & $10 . .>40$ &. $.30 \ldots$ & $<5$ \\
\hline 1200 & 150 & LRLR & $13 \ldots 16$ & $23 \ldots>40$ & $>25$ & $<8$ \\
\hline 400 & 300 & LRLR & $10 . .15$ & $22 . .>40$ & $>25$ & $<8$ \\
\hline
\end{tabular}

range resolutions. The reported work should enable future applications which combine polarimetric observations with temporal properties of the multi-channel radar echoes. The study also suggests further investigations to assess the relative influences of other radar parameters on the temporal properties of radar signals. As already indicated earlier, these parameters include: (1) the Doppler spectrum of the target, (2) the size of the impulse volume, (3) the range dependence, (4) the hydrometeor size-distribution, and (5) the signal-tonoise ratio.

\section{References}

Battan, L. J.: Radar Observation of the Atmosphere, The University of Chicago Press, 1973.

Bringi, V. N. and Chandrasekar, V.: Polarimetric Doppler weather radar Principles and Applications, Cambridge University Press, 2001.

Chandra, M., Clemens, E., Ritenberg, F., Schnabl, G., Schroth, A., and Tragl, K.: Polarimetric Meteorological Instrumentation Radar Facility, Direct and Inverse Methods in Radar Polarimetry, part 2, edited by: Boerner, W. M. et al., Kluwer Academic Publishers, 1579-1612, 1992.

Schroth, A., Chandra, M., and Meischner, P.: A C-Band Coherent Polarimetric Radar for Propagation and Cloud Physics Research, Journal Of Atmospheric And Oceanic Technology, Volume 5, 803-822, December 1988.

SIGMET Inc.: RVP8 User's Manual, http://www.sigmet.com, 2 Park Drive, Unit 1, MA01886, Westford, Massachusetts, USA, August 2004. 